\title{
Bogotá Análoga:
}

diagramas del espacio de la economía popular*

Fecha de recepción: 15 de marzo de 2016 | Fecha de aprobación: 26 de marzo de 2019 | Fecha de publicación: 30 de julio de 2019

Camilo Andrés Cifuentes-Quin

Universidad de La Salle, Colombia ORCID: 0000-0003-1470-9208 $\quad$ cacifuentes@unisalle.edu.co

Resumen Históricamente en Bogotá, las ventas ambulantes han sido consideradas como un problema público que se ha materializado en el espacio urbano y que en los últimos años se ha politizado fuertemente. El debate se ha dado en gran medida en torno a la contradicción que ha surgido entre la defensa del derecho al disfrute del espacio público y la protección del derecho al trabajo de miles de ciudadanos que ganan su sustento en la calle. En este contexto, las administraciones locales de izquierda han planteado el desarrollo de una política de creación de 'espacios análogos' como una posible solución a dicha contradicción. Este artículo presenta el contexto dentro del cual surgió esta propuesta junto con el desarrollo de una serie de prescripciones hipotéticas que invitan a reflexionar sobre las implicaciones espaciales de la hipotética implementación de una política de este tipo, y sobre las posibilidades de una metodología diagramática de análisis.

Palabras clave Bogotá, ventas ambulantes, espacio público, espacios análogos

Artículo de reflexión

Este artículo está basado en los resultados de la investigación 'Bogota: a study case on new development strategies for contemporary metropolis'. Esta investigación fue realizada, en colaboración con el Laboratorio Cresson, por Nicolas Tixier, Sandra Fiori, Jul McOisans, Céline Rouchy, Aida Assefa y Camilo Cifuentes, y fue financiada por la beca de investigación Rafael Viñoly Architects Grant. El artículo también se basa en los resultados de la contribución del autor, en colaboración con Sandra Fiori y Jul McOisans, para la exposición inaugural del concurso Minimaousse 5: "Vendeurs de rue à Bogotà" (Cité de l'architecture et du patrimoine. Paris, marzo de 2012).

Cómo citar este artículo: Cifuentes-Quin, C. A. (2019). Bogotá Análoga: diagramas del espacio de la economía popular. Cuadernos de Vivienda y Urbanismo, 12(24). https://doi.org/10.11144/Javeriana.cvu12-24.bade 


\section{Analogous Bogotá:}

Diagrams of the Popular Economy Space

Abstract In Bogotá the street sales have been historically seen as a public problem, which is materialized in the urban space and over the last years has become a strongly politicized issue. The debate has been mostly focused on the contradiction between the defense of the right to enjoy the public space and the protection of the right to work for thousands of citizens who make a livelihood from the street sales. In this context, the left-wing local administrations are proposing to develop a policy intended to create 'analogous spaces' as a potential solution to this contradiction. This article outlines the context in which they came up with such proposal. It also describes the development of a set of hypothetical provisions calling for reflection on the spatial implications of hypothetically implementing such a policy and on the potentialities of a diagram analysis methodology.

Keywords Bogotá, street sales, public space, analogous spaces

\section{Bogotá Análoga:}

\section{diagramas do espaço da economia popular}

Resumo Historicamente em Bogotá, as vendas ambulantes foram consideradas como problema público que tem se materializado no espaço urbano e que nos últimos anos se politizou fortemente. O debate tem-se dado em grande medida em torno da contradição surgida entre a defesa do direito a usufruir o espaço público e a proteção do direito ao trabalho de milhares de cidadãos que ganham seu sustento na rua. Nesse contexto, administraçóes locais de esquerda colocaram o desenvolvimento de uma política de criação de 'espaços análogos' como possível solução a essa contradição. Esse artigo apresenta o contexto dentro do que surgiu esta proposta junto com o desenvolvimento de uma série de prescriçóes hipotéticas que convidam a refletir sobre as implicaçóes espaciais da hipotética implementaçáo de uma política deste tipo, e sobre as possibilidades de uma metodologia diagramática de análise.

Palavras chave Bogotá, vendas ambulantes, espaço público, espaços análogos 


\section{Introducción: las ventas callejeras, un problema público}

Los datos hablan por sí solos: en Bogotá, el 97\% de las unidades productivas hacen parte del sector económico que el Instituto para la Economía Social (IPES) define como 'economía popular'. Tales unidades incluyen una variedad de pequeñas y medianas empresas, miles de trabajadores que trabajan por su cuenta y la economía del cuidado. Según el IPES, aunque este sector genera el $60 \%$ del empleo de la ciudad, éste se caracteriza por la precariedad de sus procesos de gestión y la calificación de su mano de obra, al igual que por el limitado acceso de los trabajadores del sector informal a desarrollos tecnológicos, a capital o a crédito (Pulecio, 2013).

Desde luego, las dinámicas generadas por este sector económico tienen importantes repercusiones en las dinámicas de la ciudad, $\mathrm{y}$, como es de esperarse, la precariedad de sus condiciones tiene una relación directa con una serie de conflictos que se materializan en el espacio urbano. Lo anterior es particularmente evidente en el caso de las ventas callejeras, que es tal vez la más visible de las actividades de la llamada economía popular.

En efecto, desde hace mucho tiempo, la venta ambulante hace parte del paisaje urbano bogotano y contribuye fuertemente a su definición. Como bien lo señala un cronista del periódico "Historia ambulante" (2002):

Eran ambulantes las aguateras que iban calle arriba calle abajo, con sus múcuras de barro, desde las pilas hasta las casas, y hoy todavía, como en aquellos primeros tiempos, se ven burros cargados con ramas de eucalipto que recorren la ciudad de norte a sur para que los clientes hagan el sahumerio de costumbre.
Al igual que en numerosas ciudades alrededor del mundo, en Bogotá los vendedores ambulantes ofrecen una variedad de menudos servicios que responden a las necesidades inmediatas que los transeúntes encuentran en sus recorridos cotidianos. Atentos a las oportunidades que ofrece la calle como lugar de trabajo, los vendedores ambulantes se instalan en puntos estratégicos, lo que genera importantes nodos de actividad, o se desplazan de acuerdo con los flujos y eventos que de manera permanente se crean y se deshacen en el espacio público.

Por lo tanto, los vendedores callejeros son protagonistas de lo que el antropólogo Manuel Delgado (2005) define como el 'ámbito de los discurrires', ese mundo emergente que se gesta en la calle, que prima sobre la estabilidad estructural que proyectan los políticos y los urbanistas, y que estudiosos de la ciudad, como Jane Jacobs, Henri Lefebvre, Issac Joseph, y el mismo Delgado, han exaltado y situado como un aspecto primordial de los fenómenos urbanos.

Pero más allá de su importancia en la definición de los ambientes de la ciudad (de sus aspectos pintorescos, de su exhibición de creatividad, y de su admirable capacidad para inventar soluciones a las necesidades de los sectores desatendidos por el Estado y relegados a la informalidad por el neoliberalismo), la actividad de los vendedores ambulantes es también la materialización en el espacio urbano de una problemática que, en el caso bogotano, ha sobrepasado la capacidad de respuesta de los diferentes gobiernos de la ciudad. Al fin y al cabo, las ventas callejeras no son sino un aspecto 
de la informalidad económica que predomina en Bogotá, un fenómeno que se ve reflejado en la precariedad del empleo y de las condiciones salariales de cientos de miles de habitantes urbanos. A estos problemas estructurales se suman los conflictos que surgen de la apropiación del espacio público para el desarrollo de actividades comerciales, entre los cuales se destacan, por un lado, la emergencia de poderes mafiosos que controlan y explotan el derecho al uso del espacio; y por el otro, la estigmatización de quienes buscan su sustento en la calle. Teniendo en cuenta lo anterior, las ventas callejeras aparecen como el reflejo de una realidad social compleja que, en el caso concreto de Bogotá, se ha politizado fuertemente desde hace más de dos décadas y ha sido objeto de debate, tanto en los círculos de decisión como en los círculos académicos nacionales e internacionales (Donovan, 2008; Hunt, 2009; Rocha, Sanchez y García, 2009; Alfonso, 2010; Berney, 2011; Galvis, 2014; Aliaga-Linares, 2018).

\section{El derecho al espacio contra el derecho al trabajo}

Con el regreso de Enrique Peñalosa al gobierno de Bogotá en 2016, la importancia del espacio público - uno de los discursos bandera del alcalde reelegido y del exalcalde, Antanas Mockus- volvió a situarse como una prioridad de la administración distrital. En consecuencia, el problema de las ventas callejeras, al igual que los discursos de la 'recuperación' del espacio público, volvieron a aparecer como un tema de intenso debate público en la ciudad ${ }^{1}$. No obstante, el protagonismo de este asunto en la agenda de los gobiernos locales no es nuevo, por el contrario, se trata de una cuestión que durante casi dos décadas ha ocupado un lugar prominente, tanto en los planes de desarrollo de la ciudad ${ }^{2}$-independientemente de la orientación política de los diferentes gobiernos- como en la agenda de los medios de comunicación y las asociaciones de comerciantes (Tixier et al., 2011). Este debate ha tenido como telón de fondo diferentes enfoques sobre las conexiones casuales de la economía informal con el crecimiento de la pobreza urbana, los cuales han sido promovidos por académicos y organismos multilaterales. Tales enfoques han preconizado desde la simplificación de la regulación de las ventas ambulantes hasta la importancia del estado como agente activo en la promoción de la igualdad laboral y del acceso de los trabajadores del sector informal al sector formal de la economía (AliagaLinares, 2018).

En gran medida, la discusión sobre la regulación de las ventas callejeras en Bogotá se ha dado en torno a la contradicción evidente que ha surgido entre la obligación de los alcaldes de defender el disfrute del espacio público como un derecho colectivo y la defensa del derecho al trabajo como un derecho fundamental de todos los ciudadanos, consagrado por la Corte Constitucional.

Así, desde la perspectiva de la defensa del espacio público ha surgido una visión de la ciudad como lugar pacificado, claramente definido y portador de un discurso único (Alcaldía Mayor de Bogotá, 1998). De esta manera ha prevalecido la idea según la cual el derecho al disfrute colectivo del espacio, por una sociedad libre de conflictos de clase (Galvis, 2014), debe primar sobre su apropiación para usos 'inapropiados', cuya prohibición o intento de regulación -interpretada de diferentes maneras por los diferentes gobiernosha sido parte de una estrategia de 'recuperación' del espacio público, promovida especialmente por los alcaldes Antanas Mockus, desde el urbanismo pedagógico, y Enrique Peñalosa, desde la intervención física del espacio urbano. 
Por esta vía se ha promovido un discurso que asocia las ventas callejeras con un uso inadecuado del espacio urbano - producto de una cultura desprovista de un concepto de 'lo público'- y con imaginarios de ilegalidad, inseguridad y desorden que han reforzado la estigmatización de quienes trabajan en la calle. De hecho, la visión del espacio urbano inherente a los discursos de 'recuperación' parece alinearse con la noción de pedestrianism, que defiende una comprensión de la calle como un espacio finito, en competencia y amenazado por intereses múltiples, cuya principal función (y la de las autoridades) debe ser garantizar el movimiento ordenado de los peatones (Blomley, 2010). En consecuencia, en lo que concierne a las ventas callejeras, las políticas de recuperación del espacio público han buscado su desaparición, sea mediante su prohibición o su relocalización.

Aunque en las políticas de recuperación del espacio público en Bogotá, iniciadas durante el primer gobierno de Enrique Peńalosa, entre 1998 y 2001, se planteó la necesidad de ofrecer alternativas laborales a los vendedores. Las acciones llevadas a cabo parecen haber privilegiado el problema del espacio sobre el problema del empleo. Como lo muestran diferentes estudios, la política de recuperación del espacio público en Bogotá no ha estado exenta de controversia y esta no siempre ha operado en sentido del mayor beneficio de los vendedores. En algunos casos documentados, las políticas de recuperación del espacio público han demostrado tener un impacto negativo en los ingresos de los vendedores, e incluso han permitido la instauración de prácticas excluyentes que contrastan con los discursos de inclusión sobre los que estas se han cimentado (Donovan, 2008; Galvis, 2014; Castro-Cabrera, 2003).

Con la llegada de diferentes mandatarios de izquierda al gobierno de la ciudad, los discursos sobre el espacio público se matizaron, lo cual dio paso a concepciones del espacio público (y de las políticas correspondientes) que corresponden a un enfoque más basado en los derechos.
Tal cambio de perspectiva ha permitido pensar la calle no sólo como el lugar para el tránsito ordenado, sino como lo que Lofland (1998) llama 'el reino de lo social'; a saber, un espacio con diferentes usos y significados, y, especialmente, como espacio para la realización, no sólo de los derechos individuales sino de los derechos colectivos. Este cambio coincidió con la resolución 772 de la Corte Constitucional en 2004, la cual juzgó que la política de prohibición violaba el derecho al trabajo de los vendedores callejeros. De este modo, entre 2004 y 2016, los gobiernos de izquierda propusieron nuevos enfoques más comprensivos que las políticas de relocalización y evicción. De este modo, también se reconoció, de manera más clara, que la cuestión de las ventas ambulantes tiene su origen en una problemática social y económica compleja que no se resuelve con su prohibición. De igual manera, se reconoció que las ventas callejeras constituyen un fenómeno que necesita, más que una solución a los conflictos que genera su presencia en el espacio público urbano, respuestas de la administración local que ofrezcan alternativas laborales a quienes viven de esta actividad y de diferentes actividades económicas informales.

En este sentido, la política orientada a la prohibición y erradicación de las ventas callejeras en el espacio público abrió paso a una serie de propuestas de regulación de las ventas callejeras que, sin dejar de promover el derecho colectivo al uso del espacio público, planteaban la necesidad de generar soluciones alternativas que permitieran a los vendedores de calle ejercer su derecho al trabajo. Este manejo, por cierto, fue interpretado por ciertos sectores como una política permisiva que habría conducido a que los vendedores "se multiplicaran" (“Vendedores ambulantes por doquier”, 2014).

Así, siguiendo una línea de acción dirigida hacia la regulación de las prácticas económicas informales, las administraciones de izquierda han intentado dar un nuevo estatus a tales prácticas a partir de su definición como actividades de la 
llamada 'economía popular'. Este giro lingüístico permite situar estas prácticas, no como un sector excluido y condenado, sino "como el espacio económico de los sectores populares que deben ser apoyados en sus potencias, sus capacidades creativas" (Pulecio, 2013, pp. 20-21).

De esta manera, en un contexto en el que el desempleo y la pobreza seguían siendo problemas estructurales del país, y en el que, además, los gobiernos locales tenían muy poco margen de maniobra en términos del diseño y aplicación de las políticas macroeconómicas capaces de atacar el problema del desempleo, durante el gobierno de Gustavo Petro se retomó la idea, formulada durante el mandato de Luis E. Garzón, de aportar desde el diseño del espacio público urbano una posible solución a la contradicción existente entre el derecho al espacio y el derecho al trabajo que afecta a miles de ciudadanos. Esta idea se materializó en un proyecto de planificación urbana presentado por el IPES en 2005, que contemplaba la creación de espacios públicos dedicados al desarrollo de actividades comerciales. Más concretamente, el proyecto planteaba la creación de mercados incorporados en lotes vacíos, pasajes o zonas con grandes flujos peatonales que pudieran albergar las actividades de los vendedores de calle. A este tipo de intervenciones se les denominó 'espacios análogos' (Alcaldía Mayor de Bogotá, 2005).

\section{Espacios Análogos}

Según el investigador Alexandre Roig, la contradicción entre el derecho al espacio y el derecho al trabajo desaparece precisamente en el momento en que los derechos dejan de pensarse como derechos individuales para ser pensados como derechos colectivos. En este sentido, Roig se pregunta “¿Cuál es la diferencia entre estar en una calle y estar en una plaza?” (Molina, Wanderlay, Roig y Correa, 2013, p. 52). Su respuesta es que la calle es un lugar de "apropiaciones privativas de pequeños espacios que compiten entre si” (p. 52), mientras que la plaza es un lugar común en el que todos los actores que participan en ese espacio tienen que definir "colectivamente el uso del espacio. Por eso es tan importante pasar de una calle a una plaza porque en una plaza se puede construir un derecho colectivo" (p. 52). En otras palabras, lo que Roig sugiere es que la economía popular adquiere sentido sólo si esta se construye como categoría política, para lo cual es crucial construir su espacio en lo común. Es justamente la construcción del espacio político de los sectores económicos populares lo que se planteó a partir de la propuesta de creación de los llamados 'espacios análogos', concebidos como lugares de carácter público, diseñados y pensados para ser ocupados por elementos de la economía popular.

Según lo estipulado en el Plan Maestro de Espacio Público, decretado en 2005, la construcción de espacios análogos debería llevarse a cabo "en las zonas centrales de la ciudad, en aquellos espacios de mayor intensidad de tránsito peatonal, en donde se han ubicado tradicionalmente las ventas callejeras” (Alcaldía Mayor de Bogotá, 2005), con el objetivo de "mejorar el aprovechamiento del suelo, elevar la calidad del parque inmobiliario en zonas amenazadas por fenómenos de deterioro urbanístico, y generar localizaciones alternativas para trabajadores informales del espacio público en proceso de legalización" (Alcaldía Mayor de Bogotá, 2005). Estas disposiciones no se tradujeron en la implementación de proyectos concretos, ni durante la administración de Luis E. Garzón, ni la de Gustavo Petro.

Aunque hay estudios que consideran que los proyectos del IPES no se diferencian radicalmente de las políticas de relocalización de las administraciones anteriores (Aliaga-Linares, 2018), y que las relocalizaciones no siempre funcionan (Donovan, 2008), la idea de crear espacios para la economía 
popular puede ser una interesante táctica para potenciar el espacio del mercado como lugar por excelencia de la vida política. Adicionalmente, la creación de este tipo de espacios podría convertirse en una estrategia de diseño urbano capaz de ofrecer una solución a las guerras por el espacio que se viven cotidianamente en las calles de Bogotá; una solución cuya efectividad depende en gran medida del reconocimiento de los sectores económicos populares como actores legítimos de la vida urbana.

Dicho lo anterior, surgen ciertas preguntas: ¿̨cuáles serían las implicaciones espaciales de establecer una estrategia para la construcción de una infraestructura de 'espacios análogos' que responda a la necesidad de generar localizaciones alternativas para las ventas callejeras? y ¿cuál es la viabilidad de generar una infraestructura de este tipo capaz de acoger al gran número de vendedores activos?

Partiendo de reconocer el potencial del espacio del mercado como hecho urbano catalizador de dinámicas socio-políticas, al igual que de la necesidad de ofrecer respuestas a las carencias de un importante sector de la población que gana su sustento en las calles, el ejercicio diagramático presentado a continuación constituye un aporte que ofrece algunas luces respecto a la viabilidad y las necesidades espaciales de la producción de una hipotética infraestructura pública de espacios análogos en Bogotá. Este ejercicio constituye, así mismo, una exploración sobre el potencial de una metodología de análisis diagramático para estudiar desde una perspectiva estadística los problemas de planificación urbana en el ámbito local.

\section{Bogotá Análoga}

Bogotá análoga es una exploración diagramática que, en referencia a las preguntas planteadas anteriormente, indaga sobre las necesidades espaciales del desarrollo de una hipotética estrategia de diseño urbano orientada a crear la infraestructura necesaria para la regularización de las ventas callejeras en el espacio público bogotano. Inspirado en el método estadístico empleado por Winy Maas y Mvrdv en trabajos como Farmax, KM3 y Metacity/Datatown, el ejercicio parte de la conversión de algunos de los datos disponibles sobre el problema en cuestión en diagramas espaciales.

El interés de este tipo de aproximación es doble. Por una parte, la aproximación diagramática constituye una herramienta que, como lo plantea Alexander (1969), permite operar simultáneamente como instrumento de análisis de un problema de diseño y como herramienta de síntesis de dicho análisis. Por otra parte, este tipo de acercamiento hace posible la exploración numérica de un problema de diseño urbano que, por sus dimensiones, parece ser mejor comprendido mediante una técnica estadística.

En el caso del análisis de la hipotética implementación de una política de creación de espacios análogos en Bogotá, lo que se pretende con este tipo de aproximación es establecer una relación entre cierta información conocida sobre las ventas callejeras y el espacio público bogotano. Lo anterior mediante la definición de lo que los autores de Metacity/Datatown llaman 'prescripciones hipotéticas' (Mvrdv, 1999). Tales prescripciones hipotéticas permiten evaluar diferentes escenarios para la creación de espacios análogos en Bogotá en correspondencia con el número estimado de vendedores activos. Cabe aclarar que este ejercicio no pretende ofrecer soluciones de diseño o lineamientos para el desarrollo de los espacios análogos, sino entender las implicaciones espaciales del problema mediante una técnica que se acerca más al análisis estadístico que al diseño espacial; esto debido a que, como ya se ha mencionado, por su magnitud, este problema sólo puede entenderse en un nivel macro mediante una técnica estadística. En este sentido, el ejercicio se 
piensa como un aporte para el debate sobre la agenda de construcción de una infraestructura de arquitectura urbana que podría potencializar el espacio de los sectores económicos populares $y$, de este modo, resolver los conflictos urbanos que genera su presencia en el espacio público.

Partamos de cuantificar el problema:

, El área urbana de Bogotá es de $307 \mathrm{~km}^{2}$.

, El área total de espacio público en la ciudad es aproximadamente de $150 \mathrm{~km}^{2}$ (Alcaldía Mayor de Bogotá, 2015).

, El área de espacio público efectivo de la ciudad es aproximadamente de $35 \mathrm{~km}^{2}$ (Alcaldía Mayor de Bogotá, 2015).

, Teniendo en cuenta los datos disponibles, el número de personas que se dedican a las ventas callejeras en Bogotá puede estimarse, aproximadamente, en 150.000. Esta es la cifra que se manejaba en 2007, según el estudio Hábitat y espacio público: el caso de los vendedores ambulantes en el espacio público físico de Bogotá (Castañeda y García, 2007). De acuerdo con los datos del IPES, en 2017 había 50.038 vendedores inscritos en el Registro Individual de Vendedores Informales (IPES, 2016), pero según Luis Ernesto Cortés, anterior director del IPES, en 2014 se estimaba que, además de los 44.000 vendedores que la ciudad tenía censados, cerca de 100.000 vendedores restantes ejercían su actividad sin afiliación a ningún programa estatal ("Vendedores ambulantes por doquier", 2014).

Si se asume que la estimación de 150.000 vendedores es acertada, y si se destinaran $3 \mathrm{~m}^{2}$ a cada uno de los vendedores activos en la ciudad, la creación de una red de espacios análogos requeriría la ocupación de alrededor de $450.000 \mathrm{~m}^{2}$ de espacio público. Esto corresponde a:
, 6,2 veces el área de los mercados distritales existentes.

, $0,15 \%$ del total del área urbana.

, $0,3 \%$ del total del área de espacio público.

, $1,5 \%$ del área de espacio público efectivo.

Los diagramas que se presentan a continuación exploran diferentes configuraciones hipotéticas para la creación de una infraestructura de espacios análogos en Bogotá. Estos diagramas parten de la necesidad de crear en el espacio público de la ciudad alrededor de $450.000 \mathrm{~m}^{2}$ de espacio de mercado para ofrecer localizaciones alternativas a los vendedores callejeros activos.

Diagrama 1. Si se pensara el desarrollo del espacio de la economía popular como una suerte de gran plaza de mercado, esta ocuparía un área aproximada de 49 hectáreas. Concebida como una matriz compuesta por unidades de $1,8 \mathrm{~m}$ x 1,8 $\mathrm{m}$, el desarrollo de este espacio ocuparía un área equivalente a un tercio del territorio de la localidad de la candelaria (figuras 1 y 2). La viabilidad de la creación de un espacio de este tipo en una zona central de la ciudad resulta bastante improbable si se tiene en cuenta que su desarrollo requeriría la creación de un espacio público cinco veces más grande que el parque del tercer milenio.

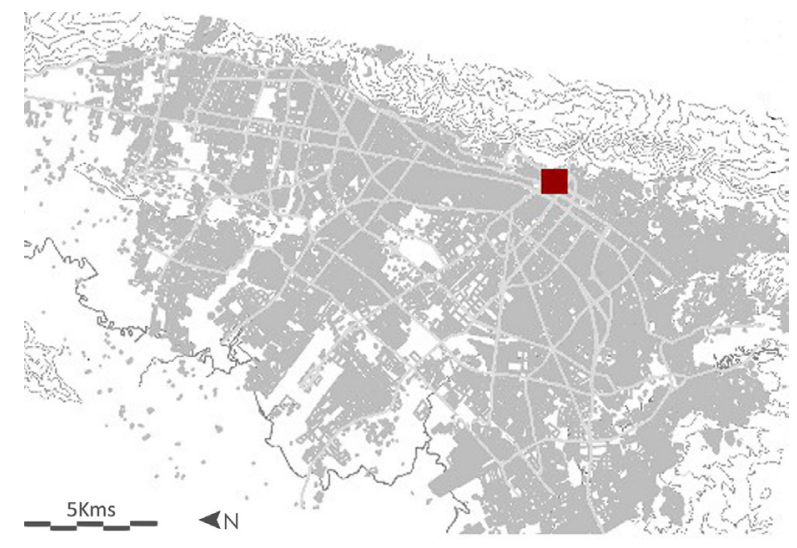

Figura 1. Localización sobre el plano de Bogotá del diagrama 1 Fuente: elaboración propia 
Diagrama 2. Si este gran espacio comercial se

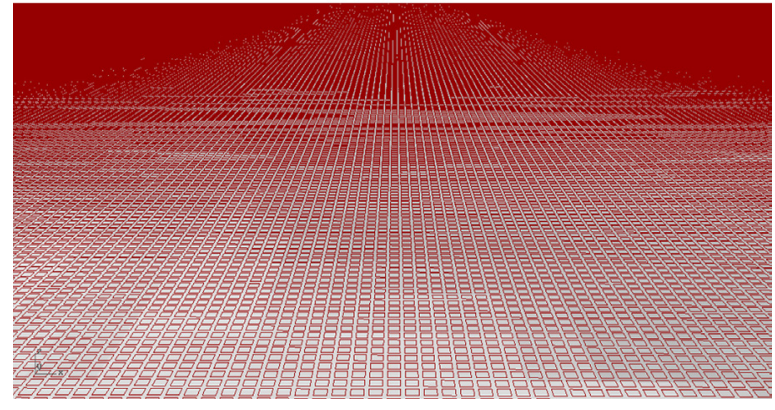

Figura 2. Diagrama 1. Detalle

Fuente: elaboración propia concibiera como un mercado lineal, como un pasaje compuesto por 10 filas de puestos de ventas alineados, su longitud sería de $42 \mathrm{~km}$. El desarrollo de esta franja equivaldría a la longitud total de tres importantes ejes viales de la ciudad, la Autopista Sur, la NQS y el tramo de la carrera séptima, entre la calle 100 y el límite de la ciudad hacia el norte (figuras 3 y 4). La realización de una solución de este tipo, que como espacio público podría ser interesante, exigiría, no obstante, reemplazar el uso como corredor vial de una parte considerable de la infraestructura de movilidad de la ciudad.

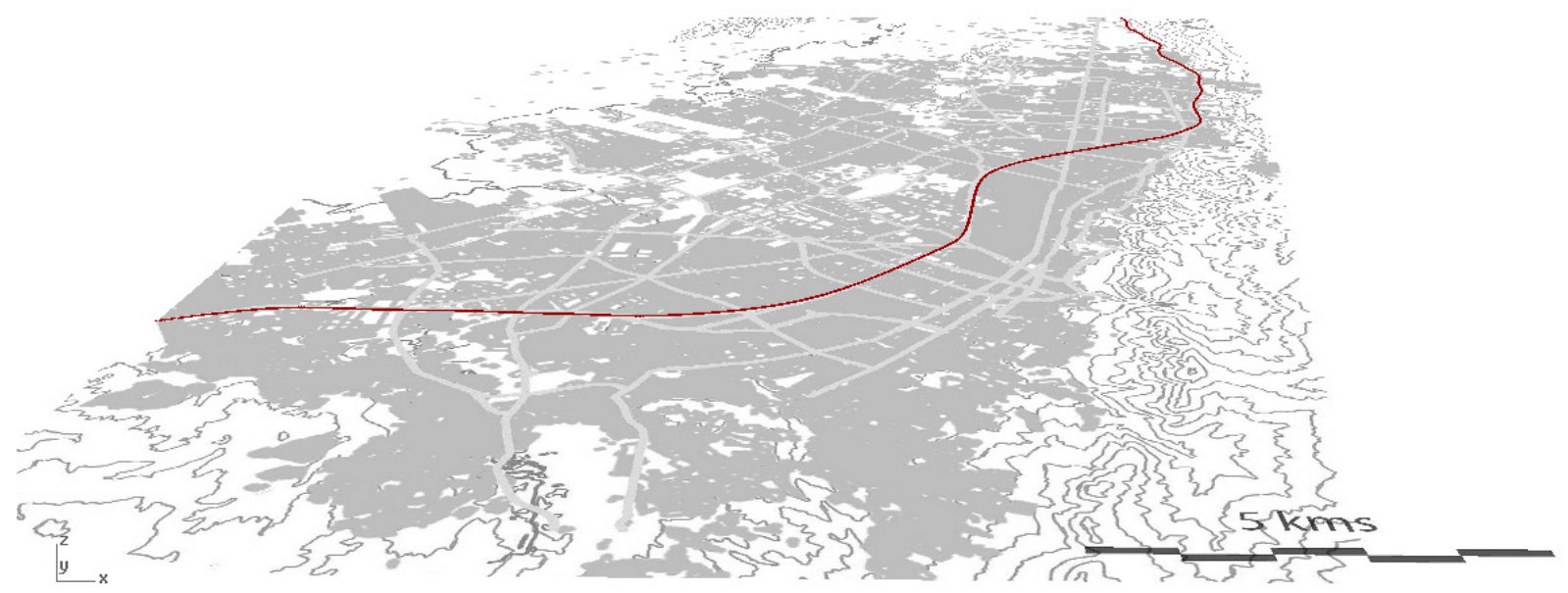

Figura 3. Localización sobre el plano de Bogotá del diagrama 2

Fuente: elaboración propia 


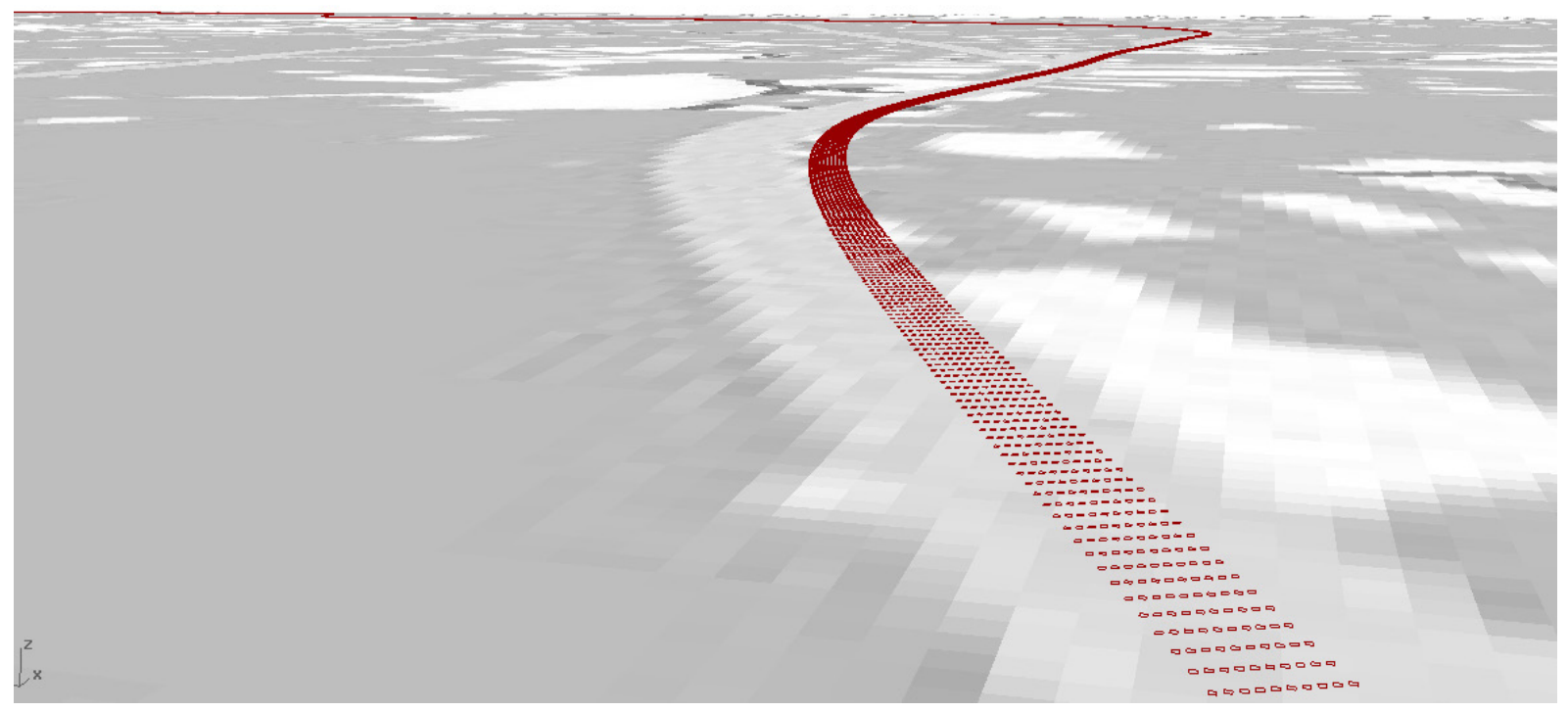

Figura 4. Diagrama 2. Detalle

Fuente: elaboración propia

Diagrama 3. Una alternativa a la solución planteada en el diagrama 2 sería que este mercado lineal sólo estuviera compuesto por dos hileras de puestos comerciales alineados, de modo que este pudiera desarrollarse sin suprimir el espacio para el transporte vehicular en los espacios

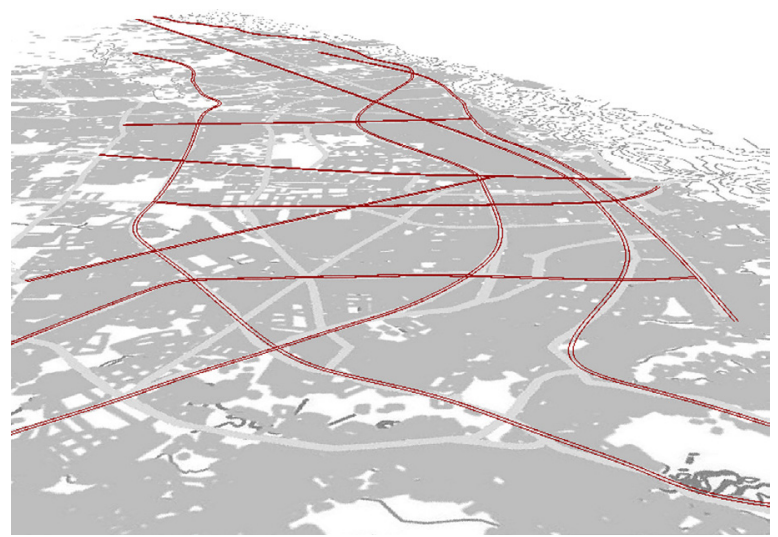

Figura 5. Mapeo sobre el plano de Bogotá del diagrama 3 Fuente: elaboración propia intervenidos. No obstante, como lo muestran las figuras 5 y 6 , una configuración de este tipo tendría una longitud de $210 \mathrm{~km}$, y requeriría adecuar y ocupar parcialmente las aceras de los principales ejes viales de la ciudad.

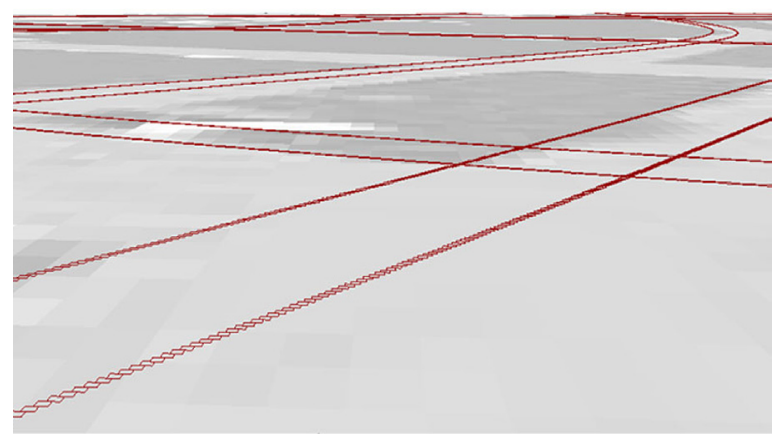

Figura 6. Diagrama 3. Detalle Fuente: elaboración propia 
Diagrama 4. En vista de las limitaciones espaciales evidenciadas por los diagramas anteriores, una opción radicalmente diferente podría ser la repartición de manera homogénea de los 150.000 puestos de venta requeridos en las localidades de mayor concentración de ventas ambulantes en la ciudad: Santa Fe, Chapinero y Usaquén (figura 7). Esto implicaría que en cualquier punto del territorio comprendido por las tres localidades habría una distancia máxima de 35 metros a un punto de venta. Si, por el contrario, estos espacios se desarrollaran únicamente en el área de espacio público existente, sería necesario localizar un puesto de venta por cada $100 \mathrm{~m}^{2}$ de espacio público efectivo. De ser viable, en términos espaciales, una solución de este tipo respondería a la necesidad de generar localizaciones alternativas para los trabajadores informales, e incluso podría hacer parte de una estrategia de renovación del espacio público. No obstante, una estrategia de atomización dificultaría la necesidad ya mencionada de construir, en parte a través del espacio, la economía popular como categoría política.

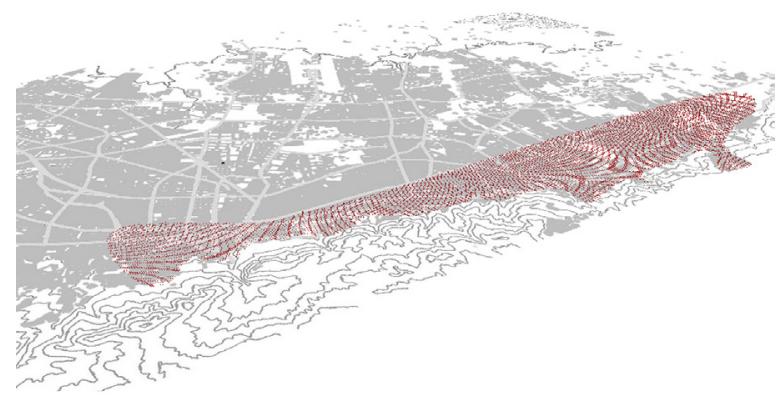

Figura 7. Localización sobre el plano de Bogotá del diagrama 4 Fuente: elaboración propia

Diagrama 5. Como alternativa a la enorme extensión de una mega-plaza y de un gran mercado lineal, o a la excesiva atomización de una estrategia de difuminación, la densificación podría ser una opción; la cual, sin embargo, iría en contra de la idea inherente al modelo de concebir los espacios análogos como plazas o pasajes comerciales. Así, si la infraestructura de espacios análogos se planteara como un edificio en altura con una base de $50 \mathrm{~m} \times 50 \mathrm{~m}$, este debería tener 461 pisos para albergar 150.000 puestos de $3 \mathrm{~m}^{2}$ (figuras 8 y 9). Con una distancia de 3,5 $\mathrm{m}$ entre placas, esta edificación tendría una altura 2,75 veces mayor a la de la estructura habitable más alta existente en el mundo.

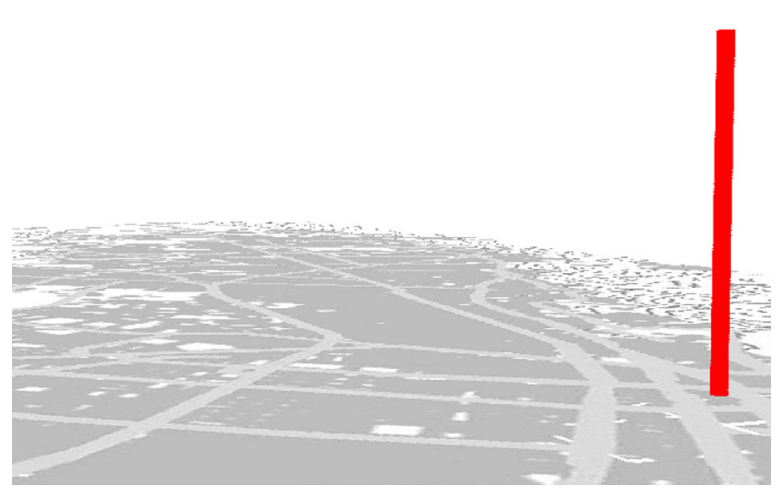

Figura 8. Localización sobre el plano de Bogotá del diagrama 5 Fuente: elaboración propia

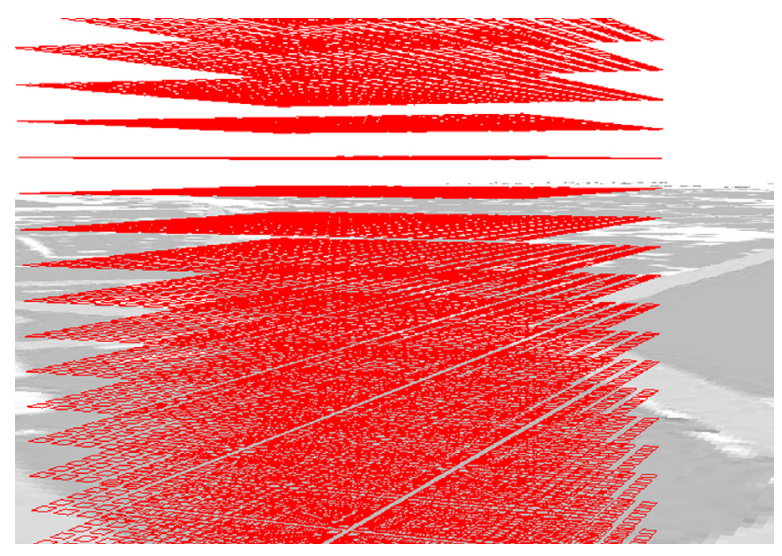

Figura 9. Diagrama 4. Detalle

Fuente: elaboración propia 
Diagrama 6. Otra alternativa de densificación podría ser que el desarrollo de los espacios análogos se articulara con el de los mercados distritales. Sin embargo, aun así, para la creación de 150.000 puestos de ventas sería necesario construir en los predios existentes 17 torres de 32 pisos de $50 \mathrm{~m} \times 50 \mathrm{~m}$ de base, cuya área total, sumada al área actual de los mercados distritales, sería de $522.000 \mathrm{~m}^{2}$ (figuras 10 y 11).

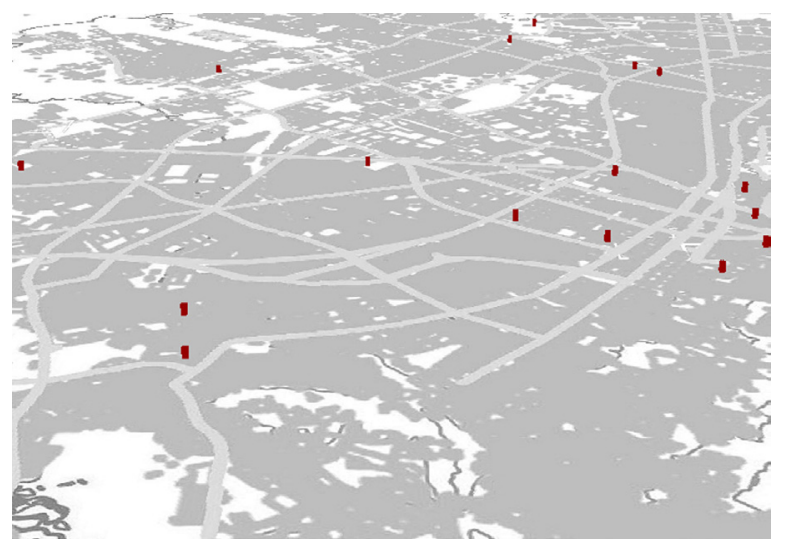

Figura 10. Localización sobre el plano de Bogotá del diagrama 6 Fuente: elaboración propia

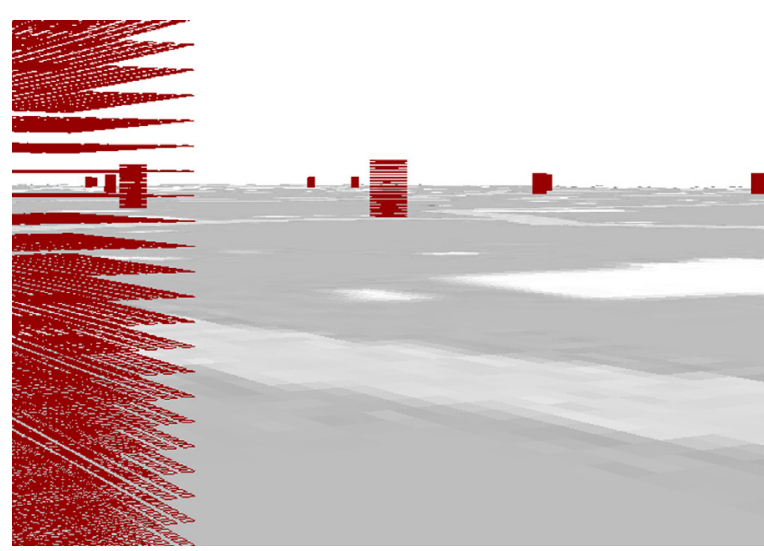

Figura 11. Diagrama 6. Detalle

Fuente: elaboración propia

Diagrama 7. En vista de la insuficiencia del área existente de los mercados distritales para acoger a los vendedores callejeros activos, y de la posible inadecuación de una tipología de torre para el desarrollo de un espacio público como el que se imagina dentro de la política de creación de espacios análogos, una alternativa sería implementar espacios de mercado en los diferentes parques zonales de la ciudad, cuya área oscila entre 1 y 10 hectáreas (figura 12). Una estrategia de este tipo requeriría crear 68 parques-mercado, cada uno de los cuales debería ser capaz de acoger una estructura de dos pisos con una huella de $5000 \mathrm{~m}^{2}$. Lo anterior cumpliría, simultáneamente, con la necesidad, identificada en el Plan Maestro de Espacio Público de 2015, de crear o fortalecer centralidades, renovar espacios públicos y generar localizaciones para los trabajadores informales, aunque también implicaría reducir, en cerca de $340.000 \mathrm{~m}^{2}$, la superficie de áreas verdes de la ciudad.

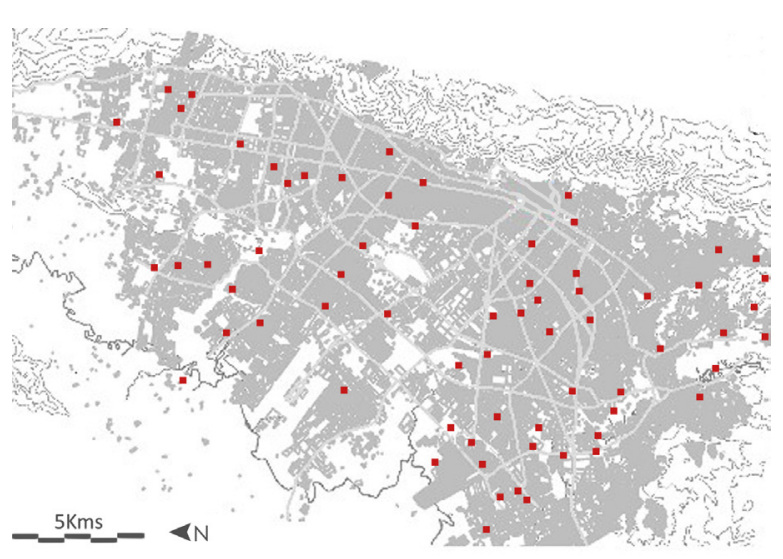

Figura 12. Localización sobre el plano de Bogotá del diagrama 7 Fuente: elaboración propia

\section{Conclusiones}

El ejercicio diagramático presentado muestra que la dimensión del problema de la informalidad económica urbana requiere respuestas complejas debido a la magnitud misma del problema. El título del ejercicio desarrollado, Bogotá Análoga, sugiere precisamente que, dada la gran cantidad de personas cuyo sustento depende de actividades económicas informales, responder a las necesidades de este sector desde la creación de espacios públicos, implicaría ejecutar proyectos de 
infraestructura que tendrían un gran impacto espacial de escala urbana en la ciudad; esto, sin mencionar otros aspectos, como el costo económico y las nuevas controversias sobre el uso del espacio que una iniciativa de este tipo podría generar. Respecto a lo anterior, es importante aclarar que una aproximación netamente estadística tiene claras limitaciones, por ejemplo, en términos de comprender las implicaciones de una intervención urbana en lo referente a una serie de variables -sociales, políticas, culturales, estéticasque el estudio estrictamente cuantitativo de un problema de diseño urbano no puede considerar.

Es por este mismo motivo que las prescripciones hipotéticas presentadas no deben pensarse como un ejercicio de diseño, ni como lineamientos para la planificación de una política de desarrollo urbano, ni como un estudio profundo del problema de la formalización en el espacio urbano de la economía popular, ni mucho menos como una crítica o una evaluación de la viabilidad de la política de espacios análogos tal como está formulada en el Plan Maestro de Espacio Público de 2005. Tales fines escapan al alcance de este trabajo. Las prescripciones hipotéticas que se exponen en este artículo no son otra cosa que una serie de anotaciones operativas sobre el problema, planteado por las anteriores administraciones distritales, de construir en el espacio público de Bogotá la infraestructura de arquitectura urbana necesaria para atender a las necesidades espaciales del sector de trabajadores informales, representado por los vendedores de calle.

Aunque el ejercicio no tiene en cuenta una serie de aspectos importantes, tales como la forma y el significado que deberían tener los espacios de la economía popular, la comprensión de las implicaciones espaciales de la realización de dicha infraestructura es clave para comprender la complejidad de la implementación de una hipotética política de creación de espacios análogos capaz de responder, mediante la construcción de infraestructura urbana, a la magnitud del problema de las ventas ambulantes. Téngase en cuenta que, a diferencia de la exploración diagramática presentada, la propuesta de creación de espacios análogos elaborada por el IPES en 2005 tan solo contemplaba la ubicación de 4000 vendedores.

Como aporte a la comprensión de un problema complejo, el diagrama aparece como una herramienta de interés. En el caso específico que concierne esta investigación, los diagramas elaborados muestran que, aunque sea sólo por sus importantes necesidades en términos de espacio, la cuestión de la hipotética creación de espacios análogos en Bogotá presenta enormes desafíos, empezando por la competencia por el espacio que la creación de una infraestructura de este tipo plantearía a otras actividades de la vida urbana, como la movilidad y la recreación al aire libre. De lo anterior se desprende que el reto de una ciudad como Bogotá no es únicamente encontrar soluciones de diseño adecuadas a las necesidades del espacio público urbano, y especialmente de los sectores económicos populares, sino garantizar los recursos y crear los instrumentos de planeación necesarios para la ejecución de este tipo de proyectos. Esto último sumado a la necesidad de construir políticas que permitan dar respuesta al problema de la informalidad del empleo, que, por su magnitud, muy difícilmente puede abordarse estrictamente como un problema de diseño urbano.

En este sentido, el ejercicio también señala la relevancia de promover, tanto en los círculos académicos como en los círculos de decisión, una reflexión seria sobre la viabilidad y las implicaciones sociales, económicas, políticas y urbanísticas de la implementación de un modelo de intervención del espacio público, el cual, si aspira a aportar una solución real en el corto plazo a las guerras por el espacio que se viven cotidianamente en las calles de la ciudad, debe ser muy ambicioso. 


\section{Referencias}

Alcaldía Mayor de Bogotá. (1998). Plan de desarrollo económico y social y de obras públicas para Santa Fe de Bogotá, D. C., 1998-2001: Por la Bogotá que queremos. Bogotá: Alcaldía Mayor de Bogotá.

Alcaldía Mayor de Bogotá. (2005). Plan Maestro de espacio público. Bogotá: Alcaldía Mayor de Bogotá.

Alcaldía Mayor de Bogotá. (2015, 15 de marzo). Radiografía del espacio público en Bogotá. Recuperado de www.bogota.gov.co

Alfonso, W. H. (2010). Politización de las ventas ambulantes en Bogotá. Revista de Arquitectura, 12(1), 4-16.

Aliaga-Linares, L. (2018). The paradoxes of Informalizing Street Trade in the Latin American City. International Journal of Sociology and Social Policy, 38(7-8), 651-672.

Alexander, C. (1969). Ensayo sobre la sintesis de la forma. Buenos Aires: Editorial Infinito.

Berney, R. (2011). Pedagogical urbanism: Creating citizen space in Bogotá, Colombia. Planning Theory, 10(1), 16-34.

Blomley, N. (2010). Rights of passage: Sidewalks and the regulation of public flow. Londres: Routledge.

Castañeda, A., y García, J. (2007). Hábitat y espacio público: el caso de los vendedores informales en el espacio público físico de Bogotá. Bogotá: Alcaldía Mayor de Bogotá.

Castro-Cabrera, M. (2003). Gestión del desplazamiento por construcción de obra pública en Bogotá.
Trabajos de grado CIDER Vol. 6. Bogotá: Universidad de Los Andes.

Delgado, M. (2005). Elogio del afuera: lo urbano como sociedad sin asiento. Arquitectos, $116(5-$ 4), 55-76.

Donovan, M. G. (2008). Informal cities and the contestation of public space: The case of Bogotás street vendors, 1988-2003. Urban Studies, 45(1), 29-51.

Galvis, J. P. (2014). Remaking Equality: Community Governance and the Politics of Exclusion in Bogota's Public Spaces. International Journal of Urban and Regional Research, 38(4), 1458-1475.

"Historia ambulante". (2002, 15 de diciembre). El tiempo. Recuperado de https://www.eltiempo. com/archivo/documento/MAM-1352701

Hunt, S. (2009). Citizenship's place: the state's creation of public space and street vendors' culture of informality in Bogota, Colombia. Environment and planning D: Society and Space, 27(2), 331-351.

Instituto para la Economía Social (IPES). (2016). Caracterización vendedores ambulantes en Bogotá. Recuperado de http://www.ipes.gov.co/images/ informes/Estudios_e_investigaciones/CARACTERIZACION_VENDEDORES_INFORMALES_EN_BOGOTA2016.pdf

Lofland, L. H. (1998). The public realm: Exploring the city's quintessential social territory. Nueva York: Routledge.

Molina, H., Wanderlay, F., Roig, A., y Correa, H. (2013, abril). El espacio público y la economía popular. Conferencia presentada en el Seminario internacional y diálogos locales. Economía popular ¿Qué es y para donde va en Bogotá? Bogotá. 
Mvrdv. (1999). Metacity Datatown. Rotterdam: 010 Publishers.

Pulecio, J. (2013, abril). La economía popular en el plan de desarrollo Bogotá Humana. Ponencia presentada en Seminario Internacional y Diálogos Locales. Economía popular ¿Qué es y para donde va en Bogotá? Bogotá.

Rocha, R., Sánchez, F., y García, L. (2009). Ventas callejeras y espacio público: efectos sobre el comercio de Bogotá. Revista Desarrollo y Sociedad, (63), 245-268.
Tixier, N, Assefa, I., Cifuentes, C., Fiori, S., Mcoisans, J., y Rouchy, C. (2011). The recuperation of public space: A closer look at Bogotá, Colombia. En N. Kaufman (ed.), Pressures and Distortions. City Dwellers as Builders and Critics: Four Views (pp. 339-433). Nueva York, Estados Unidos: The Research Program of Rafael Vinoly Architects.

"Vendedores ambulantes por doquier". (2014, 31 de mayo). Semana. Recuperado de https://www. semana.com/nacion/articulo/en-bogota-vendedores-ambulantes-por-doquier/390120-3

\footnotetext{
Por ejemplo, entre enero 1 y marzo 11 de 2016 en el periódico El tiempo se publicaron ocho artículos sobre el problema de las ventas ambulantes y las acciones de "recuperación" del espacio público puestas en marcha por el alcalde Peñalosa desde su posesión.

2 Véase al respecto: Plan de desarrollo económico y social y de obras públicas para Santa Fe de Bogotá, D.C., 1995-1998; Plan de desarrollo económico y social y de obras públicas para Santa Fe de Bogotá, D.C., 1998-2001; Plan de desarrollo económico y social y de obras públicas para Santa Fe de Bogotá, D.C., 2004-2008; y Plan de desarrollo 2012-2016. Bogotá Humana.
} 